\title{
XXXIV. On the effect of absorption on the resolving power of prism trains, and on methods of mechanically compensating this effect
}

\section{F.L.O. Wadsworth}

To cite this article: F.L.O. Wadsworth (1903) XXXIV. On the effect of absorption on the resolving power of prism trains, and on methods of mechanically compensating this effect, Philosophical Magazine Series 6, 5:27, 355-374, DOI: 10.1080/14786440309462932

To link to this article: http://dx.doi.org/10.1080/14786440309462932

巴nublished online: 15 Apr 2009.

Submit your article to this journal 주

Џ Article views: 2

Q View related articles $\square$

Citing articles: 3 View citing articles $\square$ 
more than half the true value, while it made the value of $e$ twice as great as it ought to have been.

If we know the value of $e$, we can at once deduce the number of molecules in a c.c. of gas at $0^{\circ} \mathrm{C}$. and $760 \mathrm{~mm}$. pressure. For it $\mathrm{N}$ is this number, then, since $e$ is the same as the charge on the hydrogen ion in the electrolysis of solutions,

$$
\begin{gathered}
\mathrm{N} e=1.22 \times 10^{10} \\
\text { since } \quad e=3.4 \times 10^{-10}, \quad \mathrm{~N}=3.6 \times 10^{19} \text {. }
\end{gathered}
$$

This number is well within the limits of the various determinations made by the methods of the kinetic theory. The above method for determining $\mathrm{N}$ has the advantage of not involving a knowledge of the shape of the molecules, or any assumption as to the nature of the effects produced when two molecules come into collision.

In making the experiments described in this paper, $I$ have had the help of my assistant Mr. Everett.

XXXIV. On the Effect of Absorption on the Resolving Power of Prism Trains, and on Methods of Mechanically Compensating this Effect. By F. L. O. WADsWorth**

\section{[Plate VIII.]}

T previous investigations $\dagger$ of the resolving-power of prism 1 spectroscopes, it has been generally assumed, 1st, that the illumination is uniform over the wave-front passing

* Communicated by the Author. The general investigation on resulving-power, of which the present paper forms a part, was begun nearly eight years ago, but the work has been much interrupted and delayed by other more imperative and immediate demands upon the writer's time and attention. The publication of various parts of the work has therefore been more scattered and irregular than might otherwise have been the case. Since this paper was written, Prof. Campbell, with whom I fortunately had an opportunity of discussing it, has called my attention to a paper on the same subject that was published about a year ago by Dr. Reese of the Lick Observatory Staff. On examining this paper (Astrophys. Journ. xiii, p. 199, April 1901) I find that Dr. Reese has deduced the general equation corresponding to (11) of this paper (for $b_{1}=0$ ), but has confined his investigations of the effects of absorption to the particular case where the value of $\beta$ is very small. Hence his conclusion (p. 206) "that the resolving power of the Mills Spectrograph is diminished by less than one half of one per cent. by the absorption of the prisms " is incomplete, and for that reason erroneous and misleading as a general conclusion. I have therefore allowed my own paper to stand as it was originally written, acknowledging Dr. Reese's priority of publication as above.

+ See Rayleigh, Phil. Mag. vol. viii. pp. 261-264; vol.ix. pp. 49-56; vol. xlii. p. 167 ; Enc. Brit. vol. xxiv. pp. 430-439; Schuster, ibid. vol. xxii. pp. $373-374$; also papers by the writer, Astrophysical Journal, vol. i. p. 52 ; vol. iii. pp. 176 and 321 ; vol. iv. p. 54 ; vol. vi. p. 27 ; vol. xvi. p. 1 ; Phil. Mag. vol. xliii. p. 317 . 
356 Prof. F. L. O. Wadsworth on the Effect of

through the prism-train ; 2nd, that the material of the latter is of the same temperature and refractive index throughout.

The general theoretical results agree so closely with those obtained in practice that it is certain that the error, if any, introduced by either of these assumptions must be small under usual conditions. It is not, however, a priori certain that this would be true when the absorption of the glass, or the differential temperature-change in different parts of the prisms, becomes abnormally large, as it may do in certain regions of the spectrum, and under conditions of use of the instrument such as are met with in astrophysical work. The following investigation was undertaken in order to determine the actual magnitude of the effects to be expected from large variations in absorption and optical density over the transmitted wave-front.

The most general expression for the distribution in intensity in the image of a point formed at the focal plane of a telescope is

$$
\mathrm{I}^{2}=\left[\iint \frac{i}{\lambda \rho} \sin \frac{2 \pi}{\lambda}(a t-\rho) d x d y\right]^{2}, . .
$$

where $i$ is the amplitude of vibration of any element $d x, d y$ in the wave-front : $\rho$ is the distance of this element from a point $p$ in the focal plane at which the intensity is desired.

In reducing this general expression to the form usually given * the following assumptions are made $f:-$

1st. That the amplitude of vibration is constant, $i=$ const.

2 nd. That the wave-front passing the diffracting aperture is truly spherical, i.e.,

$$
x^{2}+y^{2}+z^{2}=f^{2} .
$$

3rd. The light which unites to form the image is strictly monochromatic, i.e., $\lambda=$ const.

Under these assumptions (1) may be reduced at once to the form given by Lord Rayleigh,

$$
\begin{aligned}
\mathrm{I}^{2} & =\frac{1}{\lambda^{2} f^{2}}\left[\iint \sin \left(\frac{2 \pi \xi}{\lambda f^{\prime}}+\frac{2 \pi \eta}{\lambda f}\right) d x d y\right]^{2} \\
& +\frac{1}{\lambda^{2} f^{2}}\left[\iint \cos \left(\frac{2 \pi \xi}{\lambda f}+\frac{2 \pi \eta}{\lambda f}\right) d x d y\right]^{2}, .
\end{aligned}
$$

where $\zeta, \eta$ are the coordinates of any point in the focal-plane image.

* Rayleigh, article " Wave Theory," Enc. Brit. vol. xxiv. p. 430.

- † See also Popular Astronomy, "Problems Relating to . . Resolving Power of Telescopes," vol. v. pp. 528-536; and Astrophysical Journal, vol. xvi. pp. 266 et seq. 
Absorption on the Resolving Power of Prism Trains. 357

If' we retain $i$ as a variable function,

$$
i=\phi(x y), \text {. . . . . . . }
$$

but consider the second and third assumptions as correct, we obtain similarly

$$
\begin{aligned}
\mathrm{I}^{2} & =\frac{1}{\lambda^{2} f^{2}}\left[\iint_{0} \phi(x y) \sin \left(\frac{2 \pi \xi}{\lambda f^{\prime}}+\frac{2 \pi \eta}{\lambda f^{\prime}}\right) d x d y\right]^{2} \\
& +\frac{1}{\lambda^{2} f^{2}}\left[\int \oint \phi(x y) \cos \left(\frac{2 \pi \xi}{\lambda f^{\prime}}+\frac{2 \pi \eta}{\lambda f^{\prime}}\right) d x d y\right]^{2} .
\end{aligned}
$$

In the case of the prism spectroscope the aperture of the prism-train is generally rectangular, and if all parts of the beam of light falling on the first prism-face are of uniform intensity, the diminution in the amplitude of vibration due to the absorption of the glass of the train will be uniform along any line parallel to the refracting edge of the prisms. Along any line at right angles to this the change will be expressed by the law

$$
i=i_{0} e^{-\frac{\beta}{2}\left(l-l_{0}\right)} . \text {. . . . . . }
$$

where $i$ and $i_{0}$ are the amplitudes of vibration of the ray after traversing thicknesses of $l$ and $l_{0}$ centimetres of glass, and $\beta$ is the coefficient of absorption.

If we choose the two lines just defined as the axes of $y$ and $x$ respectively we have for $\phi(x y)$ in (3)

$$
\phi(x y)=i_{0} e^{-\mathrm{B} x} \quad \text {. . . . . . . }
$$

since the length of path, $l-l_{0}$, through any given point in the prism-train is directly proportional to $x$, the distance of that point from the central ray.

The functin $o e^{-\mathrm{B} x}$ is independent of $y$, and, for a rectangular aperture, the limits of integration for $y$ in both terms of (4) are constant. Hence we obtain at once for the distribution in intensity along the axis of $\xi$ in the focal plane

$$
\begin{aligned}
\mathrm{I}_{\xi}^{2} & =\frac{i_{0}{ }^{2} d^{2}}{\lambda^{2} f^{2}}\left[\int_{-\frac{b}{2}}^{+\frac{b}{2}} e^{-\mathrm{B} x} \sin \frac{2 \pi \xi}{\lambda f} x d x\right]^{2}+\frac{i_{0}^{2} d^{2}}{\lambda^{2} f^{2}}\left[\int_{-\frac{b}{2}}^{+\frac{b}{2}} e^{-\mathrm{B} x} \cos \frac{2 \pi \xi}{\lambda f} x d x\right]^{2} \\
& =\frac{i_{0}{ }^{2} d^{2}}{\lambda^{2} f^{2}}\left[\mathrm{C}^{2}+\mathrm{S}^{2}\right] . \text {. . . . . . . . . . . . . . . . . . . . . (7) }
\end{aligned}
$$

For convenience put

$$
\frac{2 \pi \xi}{\lambda f^{\prime}}=k \text {. }
$$

Phil. Mag. S. b. Vol. 5. No. 27. March 1903. 
Then integrating each of the two terms of (7) by parts we obtain

$$
\begin{aligned}
& \mathrm{C}=k\left(e^{\frac{\mathrm{B} b}{2}}-e^{-\frac{\mathrm{B} b}{2}}\right) \cos \frac{k b}{2}-\mathrm{B}\left(e^{\frac{\mathrm{B} b}{2}}+e^{-\frac{\mathrm{B} b}{2}}\right) \operatorname{in} \frac{k b}{2} ! \\
& \mathrm{S}=k\left(e^{\frac{\mathrm{B} b}{2}}+e^{-\frac{\mathrm{B} b}{2}}\right) \sin \frac{k b}{2}+\mathrm{B}\left(e^{\frac{\mathrm{B} b}{2}}-e^{-\frac{\mathrm{B} b}{2}}\right) \cos \frac{k b}{2}
\end{aligned},
$$

and I therefore

$$
\mathrm{I}_{\xi}^{2}=\mathrm{C}^{2}+\mathrm{S}^{2}=\frac{e^{\mathrm{B} b}+e^{-\mathrm{B} b}-2 \cos k b}{k^{2}+\mathrm{B}^{2}}
$$

Resubstituting for $k$ its value in terms of $\xi$, and also expressing $\cos k b$ in terms of $\sin \frac{k b}{2}$ we finally obtain

$$
I_{\xi}^{2}=i_{0}^{2} \frac{l^{2} d^{2}}{\lambda^{2} f^{2}} \frac{e^{\mathrm{B} b}+e^{-\mathrm{B} b}-2-4 \sin ^{2} \frac{\pi \xi b}{\lambda f^{\prime}}}{(\mathrm{B} b)^{2}+4\left(\frac{\pi \xi b}{\lambda f}\right)^{2}} . . .
$$

When the origin of coordinates is not at the centre of the horizontal aperture, and the limits of integration, $b_{1}$ and $-b_{2}$, are unequal, we obtain similarly

$$
\mathbf{1}_{\tilde{\xi}}^{2}=i_{v}{ }^{2} \frac{d^{2}}{\lambda^{2} f^{2}\left(k^{2}+\mathrm{B}^{2}\right)}\left[e^{-2 \mathrm{~B} b_{1}}+e^{2 \mathrm{~B} b_{3}}-2 \cos k\left(b_{1}+b_{2}\right) e^{-\mathrm{B}\left(b_{1}+b_{2}\right)}\right]
$$

When there is no absorption $\mathrm{B}=0$ and $i=1$, and hoth (9) and (10) reduce at once to the usual form for rectangular aperture, i.e.,

$$
I^{2}=\frac{b^{2} d^{2}}{\lambda^{2} f^{2}} \frac{\sin \frac{\pi \xi b}{\lambda f}}{\left(\frac{\pi \xi b}{\lambda f}\right)^{2}} . . . . . . .
$$

For very small valnes of $B$ the distribution in intensity is practically the same as when there is no absorption. For very large values of $\mathrm{B}$, on the other hand, the term $e^{\mathrm{B} b}$ remains the only one of importance in the numerator, and we have

$$
\mathrm{I}_{\xi}^{2}=i_{0}{ }^{2} \frac{b^{2} d^{2}}{\lambda^{2} f^{2}} \frac{e^{\mathrm{B} b}}{(\mathrm{~B} b)^{2}+4\left(\frac{\pi \xi b}{\lambda f^{\prime}}\right)^{2}}
$$


Adsorption on the Resolving Power of Prism Trains. $\quad 359$

In order better to show the transition between these two extreme cases, the values of the function $I_{\xi}^{2}$ have been computed for six intermediate values of $B$ as follows:-

$$
\begin{aligned}
& \mathrm{B} b=0 \cdot 5754, \\
& \mathrm{~B} b=1 \cdot 3862, \\
& \mathrm{~B} b=2 \cdot 1972, \\
& \mathrm{~B} b=2 \cdot 7726, \\
& \mathrm{~B} b=4 \cdot 6052, \\
& \mathrm{~B} b=7 \cdot 825 .
\end{aligned}
$$

\begin{tabular}{|c|c|c|c|c|c|c|c|}
\hline \multirow{2}{*}{$\pi \stackrel{a}{a_{0}}}$. & \multirow{2}{*}{$\mathbf{I}^{2}=\frac{\sin ^{2} x}{x^{2}}$} & \multicolumn{6}{|c|}{$\mathrm{I}_{a}^{2}$ for different values of $\mathrm{B}$ \%. } \\
\hline & & $\mathrm{B} b=-5754$ & 1.3862 & $2 \cdot 1972$ & 27726 & $4 \cdot 6052$ & 7825 \\
\hline 0.00 & 1.0000 & $1 \cdot 00: 50$ & 1.0000 & 1.0000 & $1 \cdot 0000$ & 1.0000 & 1.0000 \\
\hline $0.10 \pi$ & .9675 & 9681 & 9696 & 9739 & 9766 & -9862 & 9925 \\
\hline $0 \cdot 20 \pi$ & $• 8751$ & 8769 & 8870 & $• 8997$ & 9100 & 9436 & 745 \\
\hline $0 \cdot 30 \pi$ & $7: 368$ & 7411 & 7598 & 7880 & 8114 & 8791 & 9459 \\
\hline $0.4 \pi$ & 5754 & 5792 & $\cdot 6086$ & .6535 & 6900 & 7988 & $\cdot 9079$ \\
\hline $0.5 \pi$ & $\cdot 4053$ & 4138 & 4527 & 5132 & $562 \mathrm{~s}$ & 7102 & 8620 \\
\hline $06 \pi$ & .2545 & 2646 & •3105 & $\cdot 38 \div 6$ & +416 & $\cdot 6207$ & 8128 \\
\hline $0.7 \pi$ & $\cdot 1353$ & $\cdot 1462$ & ·1955 & -2732 & :3313 & 5351 & 7599 \\
\hline $0.8 \pi$ & 0547 & .0654 & -1140 & $\cdot 1916$ & .2558 & +4624 & $\cdot 7079$ \\
\hline $0.9 \pi$ & $\cdot 0119$ & 0217 & 0663 & $\cdot 1382$ & -1989 & +4003 & 6571 \\
\hline $10 \pi$ & .0000 & .0084 & .0464 & 1089 & $\cdot 1629$ & 3494 & $\cdot 6079$ \\
\hline $1 \cdot 1 \pi$ & • 10080 & 0146 & .04 .72 & 0968 & 1422 & 3087 & $5(21$ \\
\hline $1.2 \pi$ & $02+3$ & 0292 & 0528 & .0935 & $\cdot 1303$ & .2752 & 5194 \\
\hline $1 \cdot 3 \pi$ & 0.392 & 0428 & 0605 & 0923 & $\cdot 1222$ & $\cdot 2477$ & -4798 \\
\hline $1 \cdot 4 \pi$ & 0468 & 0497 & 01332 & -0886 & $\cdot 1139$ & .2232 & $\cdot 1432$ \\
\hline $15 \pi$ & (0) 450 & 0472 & 0587 & .0805 & $\cdot 10 \div 6$ & 2008 & 4045 \\
\hline $16 \pi$ & .0358 & .0381 & $0 \pm 86$ & .0686 & .0889 & $\cdot 1798$ & 3773 \\
\hline $1.7 \pi$ & 0209 & $\cdot 0250$ & 0358 & 0554 & .0736 & 1611 & 3495 \\
\hline $1.8 \pi$ & .0108 & .0130 & 0239 & 0434 & .0626 & 1441 & 3233 \\
\hline $1.9 \pi$ & 0027 & .0049 & $\cdot 0155$ & 0344 & 05299 & -1298 & 3002 \\
\hline $20 \pi$ & . 1000 & .0020 & 0120 & 0297 & 0462 & -1182 & -3789 \\
\hline $3 \cdot 2 \pi$ & .0072 & $\cdots$ & $\ldots$ & $\cdots$ & $0+22$ & $\cdot 1011$ & 2429 \\
\hline $24 \pi$ & .0159 & $\ldots$ & $\ldots$ & $\ldots$ & 0402 & .0885 & .2116 \\
\hline $26 \pi$ & .0136 & $\ldots$ & $\ldots$ & $\ldots$ & 0347 & .0764 & 1871 \\
\hline $2 \cdot 8 \pi$ & .0045 & $\ldots$ & $\ldots$ & $\ldots$ & .0270 & $\cdot 06.51$ & 1655 \\
\hline $30 \pi$ & .0000 & ... & ... & $\ldots$ & 0210 & 0.622 & 1470 \\
\hline $32 \pi$ & $\ldots$ & $\cdots$ & $\ldots$ & $\cdots$ & $\ldots$ & $\cdot 049$ & ' 1317 \\
\hline $3 \%=$ & $\ldots$ & $\ldots$ & $\ldots$ & $\ldots$ & $\ldots$ & 043 & $\cdot 1169$ \\
\hline $40 \pi$ & $\ldots$ & $\cdots$ & $\cdots$ & $\ldots$ & $\ldots$ & $\ldots$ & .0888 \\
\hline $50 \pi$ & $\ldots$ & $\ldots$ & $\ldots$ & $\ldots$ & $\ldots$ & $\ldots$ & 0582 \\
\hline $60 \pi$ & 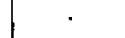 & $\cdots$ & $\ldots$ & $\cdots$ & $\ldots$ & $\ldots$ & $\cdot 0413$ \\
\hline
\end{tabular}

In these computations the quantity $i_{0}{ }^{2} \cdot \frac{l^{2} d^{2}}{\lambda^{3} f^{2}}$, whose value is determined simply by the intensity of illumination in the incident wave-front and by the dimensions of the instrument,

TABLe I. 
is so chosen that the intensity $I_{\xi}^{2}$ at the centre of the diffraction image, $\xi=0$, is the same (unity) in each case. The abscissæ are expressed in terms of the angular coordinates $\frac{\alpha}{\alpha_{0}}$ which are determined by the relations

and therefore

$$
\alpha=\frac{\xi}{f}, \quad \alpha_{0}=\frac{\lambda}{l},
$$

$$
\frac{\pi \xi l}{\lambda f}=\pi \frac{\alpha}{\alpha_{0}}=r . . . . . .
$$

The comparative values of $\mathrm{I}_{a}^{2}$ for values of a ranging from 0 to $6 \alpha_{0}$, and for the values of $\mathrm{B} b$ given above, are tabulated in columns 3, 4, 5, 6, 7, and 8 of Table I. (p. 359) and plotted as full-line curves in fig. 1. (Pl. V.LII.). For comparison the value of $\mathrm{I}^{2}$ for $\mathrm{B}=0$ is also tabulated in column 2 of this table, and plotted as a dotted curve in fig. 1 .

An inspection of this table, or the corresponding curves of fig. 1 , shows that the effect of inereasing absorption is to change the form of the curve $\mathbf{I}^{2}=f(\alpha)$, 1st, by gradually obliterating the points of maxima and minima on each side of the centre, and, 2nd, by gradually increasing its apparent width $\mathrm{mm}$. We have to consider this change in form $(a)$ on the resolving-power of the instrument, $(b)$ on the visual or photographic appearance of the spectral lines.

(a) The visual criterion for resolving-power is that two lines of equal intensity may be considered as definitely "resolved" when the illumination at the centre of their superimposed diffraction images is not more than 0.81 of the illumination at the centres of the images themselves. In the case of an instrument unaffected by absorption (equation 11 ), this corresponds to a separation of the two lines by an amount

as defined by (14).

$$
\sigma_{0}=\alpha_{0}=\frac{\lambda}{b}
$$

In the case of two equal lines separated by an angular interval $\sigma$ the relative intensities in the physical inage of the donble source at the points corresponding to the centres of the lines themselves and a point midway between them will be

$$
\frac{\mathrm{I}_{m}^{2}}{\mathrm{I}_{c}^{2}}=\frac{2 \mathrm{I}_{\sigma / 2}^{2}}{\mathrm{I}_{0}^{2}+\mathrm{I}_{\sigma}^{2}}, \quad \text {. . . . . }
$$


Atsorption on the Resolving Power of Prism Trains. 361 and the lines will be resolved or not according as $I_{m}^{2}$ is less or greater than $0.81 \mathrm{I}_{c}^{2}$.

Applying this criterion to the case of the images $I_{\alpha}^{2}$ shown in fig. 1 , we find for a constant separation $\sigma=\alpha_{0}$ the following values of $\frac{\mathrm{I}_{m b}^{2}}{\mathrm{I}_{c}^{2}}$ :-

For

$$
\begin{aligned}
& \mathrm{B} B=-5754: \frac{\mathrm{I}_{m}^{2}}{\mathrm{I}_{c}^{2}}=\frac{8 \cdot 276}{1.0084}=\cdot 82+ \\
& \mathrm{B} b=1.3862: \frac{\mathrm{I}_{m}^{2}}{\mathrm{I}_{c}^{2}}=\frac{.9054}{1 \cdot 0464}==.86+ \\
& B b=2 \cdot 1972: \frac{\mathrm{I}_{m}^{2}}{\mathrm{I}_{c}^{2}}=\frac{1 \cdot 0264}{1 \cdot 1089}=\cdot 92+ \\
& \mathrm{B} b=2 \cdot 7726: \frac{\mathrm{I}_{m}^{2}}{\mathrm{l}^{2}}=\frac{1 \cdot 1256}{1 \cdot 1629}=\cdot 97- \\
& \mathrm{B} l=4 \cdot 60.52: \frac{\mathrm{I}_{m}^{2}}{\mathrm{I}_{c}^{2}}=\frac{1 \cdot 4204}{1 \cdot 3494}=1 \cdot 05+ \\
& B l=7.825): \quad \frac{I_{m}^{2}}{I_{c}^{2}}=\frac{1 \cdot 924}{1 \cdot 608}=1 \cdot 07+
\end{aligned}
$$

which shows that the effect of absorption is to decrease the resolving-power of the instrument by an amount depending both on the value of $B$ and of $b$.

To find the separation necessary for resolution we must select a value of $\sigma$ such that

If we put

$$
\frac{2 I_{\sigma / 2}^{2}}{I_{0}^{2}+I_{\sigma}^{2}}=0 \cdot 81 . . . \quad . \quad .
$$

and

$$
\begin{aligned}
& e^{\mathrm{B} b}+e^{-\mathrm{B} b}-2=4 \mathrm{D}, \\
& \left(\mathrm{B}^{\prime} b\right)^{2}=4 \mathrm{~F}^{2},
\end{aligned}
$$

$$
\frac{\pi}{\alpha_{0}} \cdot \frac{\sigma}{2}=x,
$$

we obtain from (9) and (15)

$$
\left(\mathrm{F}^{2}+x^{2}\right)\left[\frac{\mathrm{D}}{\mathrm{F}^{2}}+\frac{\mathrm{D}+\sin ^{2} 2 u^{2}}{\mathrm{~F}^{2}+(2 x)^{2}}\right] \cong 2 \cdot \overline{5}\left(\mathrm{~F}^{2}+\sin ^{2} \cdot x\right), .
$$


from which we can determine $x$ and $\sigma$ necessary for resolution for any given values of $\mathrm{B} b$. We thus obtain

$$
\text { For } \begin{aligned}
\mathrm{B} b=\cdot 5754 & \sigma=1 \cdot 007 \alpha_{0} \\
\mathrm{~B} b=1 \cdot 3862 & \sigma=1 \cdot 04 \alpha_{0} \\
\mathrm{~B} b=2 \cdot 1972 & \sigma=1 \cdot 11 \alpha_{0} \\
\mathrm{~B} b=2 \cdot 7726 & \sigma=1 \cdot 18 a_{0} \\
\mathrm{~B} b=4 \cdot 6052 & \sigma=1 \cdot 54 \alpha_{0} \\
\mathrm{~B} b=7.825 & \sigma=2 \cdot 56 \alpha_{0}
\end{aligned}
$$

These relative values of $\mathrm{B} b$ and $\sigma$ are plotted (circles) in fig. 2 (Pl. VIII.). As is there shown, the relation between these quantities can be closely represented by the dotted curve, the equation of which is

$$
\sigma=\alpha_{0}\left\{1+\cdot 0253(\mathrm{~B} b)^{2}\right\} \text {. . . . . }
$$

an expression which is simpler and more convenient for computation than the more exact formula (16).

An inspection of the curve in fig. 2 shows that for small values of $\mathrm{B} b$, i.e. for small absorptions, the actual resolvingpower of the instrument $\sigma$ is very nearly the same as the theoretical resolving-power $\alpha_{0}$. For large values, however, it is very considerably reduced; nearly one-third for a value of $\mathrm{B} b$ equal to 4 .

To obtain a better idea of the physical conditions corresponding to the different values of $\mathrm{B} b$ considered above, it is desirable to express these values in terms of the intensities at the centre and edges of the transmitted wave-front. We have from (5) and (b)

$$
\stackrel{i}{\ddot{i}_{0}}=e^{-\mathrm{B} x} \text {. }
$$

Since the intensities at any two points are proportional to the square of the amplitudes of vibration, we have for the centre of the transmitted wave-front $x=0$, and the edge $x=\frac{h}{2}$, the ratio

$$
\frac{i_{e}^{2}}{i_{v}^{2}}=e^{\mp \mathbf{B} u}
$$

and therefore for the different values of $\mathrm{B} b$ already considered 
Absorption on the Resolving Power of Prism Trains. 363

$$
\begin{aligned}
& \frac{i_{\epsilon}{ }^{2}}{i_{0}{ }^{2}}=\frac{9}{16}=\left(\frac{3}{4}\right)^{2} \text { for } \mathrm{B} b=\cdot 5754 \\
& \frac{i_{\epsilon}{ }^{2}}{i_{0}^{2}}=\frac{1}{4}=\left(\frac{1}{2}\right)^{2} \quad, \quad=1.3862 \\
& \frac{i_{\epsilon}{ }^{2}}{i_{0}{ }^{2}}=\frac{1}{9}=\left(\frac{1}{3}\right)^{2} \quad, \quad=2.1972 \\
& \frac{i_{\epsilon}{ }^{2}}{i_{0}^{2}}=\frac{1}{16}=\left(\frac{1}{4}\right)^{2} \quad, \quad=2.7726 \\
& \frac{i_{\epsilon}{ }^{2}}{i_{0}{ }^{2}}=\frac{1}{100}=\left(\frac{1}{10}\right)^{2}, \quad=4.6052 \\
& \frac{i_{\epsilon}{ }^{2}}{i_{i_{0}}{ }^{2}}=\frac{1}{2500}=\left(\frac{1}{50}\right)^{2}, \quad=7.825
\end{aligned}
$$

or for the extreme cases the intensity at the edge of the beam traversing the base of the prism-train is fifty-six per cent. and one twenty-fifth of one per cent. respectively, of the intensity at the centre.

To find the relation between the quantities $\mathrm{B} b, \frac{i_{\varepsilon}{ }^{2}}{i_{0}{ }^{2}}$, and $\beta$, the coefficient of absorption of the glass, we have similarly from (5) and (6)

$$
\mathrm{B} x=\frac{\beta}{2}\left(l-l_{0}\right) .
$$

Also from the geometry of the prism-train

$$
\frac{x}{l_{1}-l_{0}}=\frac{\sqrt{1-n^{2} \sin ^{2} \phi / 2}}{2 \sin \phi / 2},
$$

for a single prism of refracting angle $\phi$ placed at minimum deviation. For $\mathrm{N}$ similar prisms placed en train we have similarly

$$
\frac{x}{l-l_{0}}=\frac{x}{N\left(l_{1}-l_{0}\right)}=\frac{\sqrt{1-n^{2} \sin ^{2} \phi / 2}}{2 \mathrm{~N} \sin \phi / 2},
$$

and therefore in general

$$
B=\beta N \frac{\sin \phi / 2}{\sqrt{1-n^{2} \sin ^{2} \phi / 2}}, \ldots
$$

from which we can determine the corresponding values of $\mathrm{B} b$ and $\beta$ for any given spectroseope. Some examples may be of interest.

For the Bruce spectrograph of the Yerkes Observatory, 
which is one of the largest and most recently constructed instruments of this class, we have for $b, \phi, \hat{\mathrm{N}}$, and $n$ the following values* :-

$$
\begin{aligned}
b= & 5 \cdot 1 \mathrm{cms} . \\
\phi= & 63^{\circ} 35^{\prime} \text { (mean value). } \\
N=3 . & \begin{cases}1 \cdot 653 \text { for } \lambda=5500 \dagger \\
1 \cdot 678, & \lambda=4300 \\
1 \cdot 693, & , \lambda=3900 \dagger .\end{cases}
\end{aligned}
$$

Introducing these values and solving for $\mathrm{B} b$ we find

$$
\mathrm{B} b=\beta \times \begin{cases}16 \cdot 4 \text { for } \lambda=5500 \\ 17 \cdot 2, \lambda=4300 \\ 17 \cdot 8, \lambda=3900\end{cases}
$$

The absorption of the glass of which this prism-train is composed $\left(0^{*} 102\right)$ has been determined by Vögel and Müllerł. The values of $\beta$ computed from their results are as follows:-

$$
\begin{aligned}
& \text { For wave-length } 5500, \quad \beta=.021 \\
& \text {, }, \quad 4300, \quad \beta=\cdot 071 \\
& \text { " } \quad 3900, \quad \beta=\cdot 37
\end{aligned}
$$

For the visual region of the spectrum, therefore, the value of $\mathrm{B} b$ for this spectroscope is about $\cdot 34$, and the corresponding value of $\sigma$, as given by (18), is less than $1.003 \alpha_{0}$, i.e. the resolving-power is diminished less than one-third of one per cent. by the result of absorption.

In the photographic region of the spectrum, however, the case is quite different. At wave-length $\lambda=4300$, which is about the middle of the photographic region, the value of $\mathrm{B} b$ for the Bruce spectroscope is $17 \cdot 2 \times 071=1 \cdot 22$, and the corresponding value of $\sigma$ is $1.04, i . t$. the resolving-power at this point in the spectrum is reduced about four per cent. by the result of absorption. At wave-length $\lambda=3900$ the value of $\mathrm{B} b$ is 6.59 , the value of $\sigma$ is $2 \cdot 10 \alpha_{0}$, and the resolvingpower is therefore diminished by more than fifty per cent.

For prism spectroscopes of larger size or greater resolvingpower than the one above considered the effect of absorption may become serious, even in the visible part of the spectrum,

* "The Bruce Spectroscope of the Yerkes Observatory," E. B. Frost, Astrophysical Journal, vol, xv. pp. 12-17, Jan. 1902.

+ Estimated by exterpolation from the values given by Frost.

f Astrophysical Journal, vol. v. p. 82 (1897). 
Absorption on the Resolving Power of Prism Trains. 365 particularly if a denser glass than 0.102 is used. Prism spectroscopes have been constructed having an aperture of from $2.2 \mathrm{cms}$. to $2.5 \mathrm{cms}$., and prism-trains of from 10 to 13 dense flint prisms*. These prism-trains have been constructed of very dense flint-glass whose coefficients of absorption are probably considerably higher than those for $0 \cdot 102$, which is unusually white and transparent. Assume the coefficients to be only 33 per cent. (1/3) larger. Then, since the ratio $\mathrm{B} b \div \beta$ is roughly only one-half as large for the Bruce instrument as for the Young and Grubb instruments, we have for the latter:

In the visible spectrum

$$
\mathrm{B} b=1 \cdot 00 \quad \sigma=1 \cdot 025 \alpha_{0} .
$$

In the photographic region

$$
\begin{array}{lll}
\lambda=4300 & \mathrm{~B} b \cong 3 \cdot 7 & \sigma=1.35 \alpha_{0} \\
\lambda=3900 & \mathrm{~B} b \cong 20 \cdot 0 & \sigma=11.0 \quad \alpha_{1 .}
\end{array}
$$

In this last case the resolving-power of the spectroscope is reduced more than 90 per cent. by absorption in the neighbourhood of the $\mathrm{H}$ and $\mathrm{K}$ lines.

It is evident from these results that it is useless to increase the theoretical resolving-power $r$ of our prism spectroscopes beyond a certain point. For every value of $\beta$ there will be a certain limiting value of $\alpha_{0}$, and hence of $r$, for which the practical resolving-power of the instrument as affected by absorption (which we will call $\mathbf{R}_{\beta}$ ) will be a maximum. To find this value we must transform equations (18) and (19) so that all variables are expressed in terms of $r$ and $R_{\beta}$.

From the well-known relation

$$
r^{2}=\frac{m}{\alpha_{0}}=\frac{\lambda}{\Delta \lambda}
$$

we have obviously

$$
\frac{\mathrm{R}_{\beta}}{\gamma}=\frac{\alpha_{0}}{\sigma}
$$

We have also the relation

$$
\mathrm{N} b \frac{\sin \phi / 2}{\sqrt{1-n^{2} \sin ^{2} \phi / 2}}=\frac{r}{2} \cdot \frac{d \lambda}{d n} \dagger . . .
$$

* Young, 'Nature,' vol. iii. p. 110; Grubb, Monthly Notices R. A.S. vol. xxxi. pp. 36-38.

+ See Astrophysical Journal, vol, i. p. 55 (1895). 
From (18), (19), and (20) we then obtain at once

$$
\mathrm{K}_{\beta}=\frac{r}{1+\cdot 0063\left(\beta r \frac{d \lambda}{d n}\right)^{2}}, \quad . \quad . \quad .
$$

which is similar in general form to the expression for the purity of the spectrum with slits of tinite width*.

To find the value of $r$ for which $R_{\beta}$ is a maxinum we have

$$
{ }_{d r}^{d} \mathrm{R}_{\beta}=\frac{1-\cdot 0063\left(\beta \frac{d \lambda}{d n} r\right)^{2}}{\left\{1+\cdot 0063\left(\beta \frac{d \lambda}{d n} r\right)^{2}\right\}^{2}}=0, \quad .
$$

or for $\mathrm{R}_{\beta}=\max$.

$$
r_{m}=12 \cdot 59 \frac{1}{\beta} \cdot \frac{d n}{d \lambda}, \quad \text {. . . . }
$$

and for the corresponding maximum value of $R_{\beta}$

$$
\mathrm{R}_{\beta}(\max .)=6 \cdot 29 \frac{1}{\beta} \cdot \frac{d n}{d \lambda}=\frac{1}{2} r_{m}, . . .
$$

that is, the maximum practical resolving-power that can be attained with a prism spectroscope is one-half what we could attain with the same spectroscope if there were no absorption.

For the flint glass 0.102 which we have been considering, the value of the ratio

$$
\frac{d n}{d \lambda}
$$

for wave-length $\lambda=3900$ is about 4250 . The value of $\beta$ for the same region is, as we have seen, about $\cdot 37$. Hence for this glass we have

for

$$
\begin{aligned}
\mathrm{R}_{\beta}(\max ) & \simeq 72000, \\
r_{m} & \simeq 144000 .
\end{aligned}
$$

The relation between $r$ and $\mathrm{R}_{\beta}$, as given by (21), is tabulated in Table II. and plotted in Curve 1 of fig. 3 (Pl. VIII.). It will be seen that the increase in $R_{\beta}$ is very small (less than 7 per cent. of the maximum) between the points $r=100000$ and $r_{m}=144000$; and it would therefore in general be inadvisable to go beyond the first point, if we proposed to work in the neighbourhood of the $\mathrm{H}$ and $\mathrm{K}$ lines with a prism spectroscope of dense flint glass. The theoretical resolving-power of the Bruce instrument for this region is about $153000,\left\{\frac{d n}{d \lambda}\left(t_{1}-t_{2}\right)\right\}$, and is therefore

\footnotetext{
* See Phil. Mag. vol, xliii. pp. 333-339.
} 
Alsorption on the Resolving Power of Prism Trains. 367

TABLE II.

\begin{tabular}{|c|c|c|c|}
\hline & & \multicolumn{2}{|c|}{$\mathrm{R}_{\boldsymbol{\beta}}$} \\
& & \multicolumn{2}{c|}{} \\
\cline { 2 - 4 } & II. & III. & IV. \\
\hline 25,000 & 24375 & & \\
50,000 & 44680 & 48765 & 49275 \\
75,000 & 59152 & & \\
100,000 & 67750 & 90830 & 95970 \\
125,000 & 71675 & & \\
150,000 & 72420 & 129290 & 137070 \\
200,000 & 668880 & 142460 & 169820 \\
300,000 & 56760 & 157140 & 217830 \\
400,000 & 46440 & 152720 & 239440 \\
500,000 & 38750 & 141850 & 244200 \\
600,000 & 33060 & 129420 & 239160 \\
800,000 & 25440 & 107200 & 217280 \\
1000,000 & $\cdots \cdots$ & 90100 & 192700 \\
& & & \\
\hline
\end{tabular}

considerably larger than can be used to best advantage. The Young spectroscope has a theoreticnl resolving-power $r$ of nearly 300000 in the same region of the spectrum, but as will be seen from the Table or the Curve the effect of absorption cuts down the practical resolving-power to about 57000 , actually less than that which an instrument of only one-fourth the size would possess.

It is at once evident from these results that if high-power prisin spectroscopes are to be used in the investigation of the photographic region of the spectrum, the use of the extra dense flint glass, so commonly employed in the past, must be avoided, not only on the score of light-efficiency, but, as now appears, on the score of photographic resolving-power and purity as well. The use of lighter flint reduces the theoretical resolving-power, $r$, of any given prism-train by decreasing the value of the dispersion-coefficient $\frac{d n}{d \lambda}$; but this may be easily and even advantageously compensated by increasing the refracting angle $\phi$ of the prisms. The advantages of this latter construction on the score both of light-efficiency and economy of material were pointed out by the writer several years ago*, and it has been adopted in ail the large spectroscopes recently constructed at Allegheny $\dagger$.

* Astrophysical Journal, vol, ii. p. 264 (1895).

+ Among others may be mentioned the spectroscope of the Philadelphia Observaiory, the spectroscope of the Lowell Observatory, and the spectroheliograph of the Philadelphia Observatory (just completed). Prof. Lord, of the Emerson McMillen Observatory, has also recently replaced the battery of dense flint prisms first constructed for his star spectroscope with one of light flint; and I believe Dr. Gill, of the Cape Observatory, is likewise considering this change. 
To show the advantage of the lighter glasses on the score of photographic purity the values of $R_{\beta}$ have also been computed for the dense flint, 0.93 , and the light flint, 0.340 , of Schott's table. The constants $n, \beta$, and $\frac{d n}{d \lambda}$ for ware-length 3900 for these two glasses are as follows:-

$$
\begin{aligned}
& 0.93 \quad n_{3900}=1 \cdot 66 ; \quad \frac{d n}{d \lambda}=3500 ; \quad \beta=14 \\
& 0.3 \pm 0 \quad n_{3900}=1.61 ; \quad \frac{d n}{d \bar{\lambda}}=3100 ; \quad \beta \simeq .08 .
\end{aligned}
$$

The values of $R_{\beta}$ for these values of $\frac{d n}{d \lambda}$ and $\beta$ are tabulated in columns III. and IV. of Table II., and plotted as curres II. and III. of fig. 3 (Pl. VIII.). The points of maximum for these curves are found at

$$
\begin{aligned}
& r_{m}=315000 \text { for glass } 0.93, \\
& r_{m} \cong 488000, \quad, 0 \cdot 340,
\end{aligned}
$$

and the corresponding values of $R_{3}$, (max.) are

$$
\begin{array}{lll}
1.57000 & \text { for } & 0.93, \\
244000 & \text { for } & 0.340 .
\end{array}
$$

As in the preceding case the form of the curves is such that it is not desirable to go to the extreme maximum values of of $R_{\beta}$, but to stop at point corresponding to $r \simeq 200000$ and $\cong 350000$ respectively.

For the visible portion of the spectrum the values of $n, \frac{d n}{d \lambda}$, and $\beta$ for the first glass considered, $0 \cdot 102$, are as follow:-

$$
\begin{aligned}
& n=1 \cdot 653 ; \quad \frac{d n}{d \lambda} \cong 1150, \quad \beta=\cdot 021 . \\
\therefore & \beta \frac{d \lambda}{d n}=\cdot 000018 ; \\
& r_{m} \cong 740000 \\
& R_{\beta} \text { (max.) } \cong 370000 .
\end{aligned}
$$

The above results show that while the Bruce spectroscope is quite efficient for the visible part of the spectrum, it is quite otherwise for the photographic region in the neighbourhood of the $\mathrm{H}$ and $\mathrm{K}$ lines. Such an instrument wonld not be well suited, therefore, for solar work, particularly of a spectroheliographic nature. 
Absorption on the Resolving Power of Prism Trains. $\quad \mathbf{3 6 9}$

For regions of the spectrum lying below wave-length 3900 , materials having a smaller coefficient of absorption than light flint or even crown-glass must be used. In the case of glass, indeed, we soon reach a limit beyond which a further reduction in density will be actually disadvantageous. As will be seen from ( 21$)$ and (23) we reach an absolute maximum for $\mathbf{R}_{\beta}$ when the quantity

$$
\beta \frac{d \lambda}{d n}
$$

becomes an absolute minimum. For the various flint glasses already considered, the absorption-coefficient $\beta$ decreases more rapidly than the dispersion-coefficient $\frac{d n}{d \lambda}$; hence the quotient of these two coefficients decreases with diminishing index of refraction.

But as we pass from the flints to the crowns the reverse takes place, and the value of $\beta$ decreases less rapidly than the dispersion. Hence we soon reach a point at which the quotient

$$
\beta \frac{d \lambda}{d n}
$$

is an absolute minimum for glass. Thus for the crown 0.203 examined by Vogel we have in the neighbourhood of the $\mathrm{H}$ line

$$
n_{3900} \cong 1.532, \quad \frac{d n}{d \lambda} \cong 1650, \text { and } \beta \simeq 054 .
$$

Hence for crown $0 \cdot 203$

$$
\beta \frac{d \lambda}{d n}=0.000032,
$$

which is nearly 25 per cent. larger than the corresponding quantity $(0 \cdot 000026)$ for the light flint 0.340 . Hence, in spite of its diminished absorption crown-glass would not be as good a material to use for the prisms as light flint.

We might perhaps find a flint for which the quotient of the two coefficients is somewhat less than in the case of $0 \cdot 340$. It is, however, safe to say that the value of this quotient cannot be much reduced, and that the maximum practical resolving-power which we can attain in spectroscopes with glass prisms will not exceed 250,000 units in the photographic region of the spectrum, nor 400,000 in the visual region.

In order to attain still higher resolving-powers we must use other materials than glass. Of those available for this 
purpose quartz, rock-salt, and fluorite are among the best. Unfortumately I have not been able to find any reliable data on the coefficients of absorption of these materials for different wave-lengths. Although they are considerably less than those for glass for both very short and very long wave-lengths, they are sufficiently large to exercise an appreciable effect on the resolving-power in these ultra-visible portions of the spectrum. It is also necessary to remember that certain kinds of glass, as well as other materials of which prisms may be constructed, frequently show strong selective absorption; and certain regions even in the visible part of the spectrum where this occurs may exhibit a lack of apparent definition in consequence. In fact, in all discussions involving questions of resolving-power, the possibility of the effects of large local or general absorptions must be kept in mind.

(b) The second effect of absorption which we have to consider is that on the general form and apparent "width " of the individual spectral lines. This effect is in many respects more pronounced and striking than that on the resolving power just considered; for as will be seen by an inspection of fig. 1 (Pl. VIII.), the apparent width is increased in a much greater ratio than the resolving-power is diminished by increased ahsorption.

As long as there is a well-marked minimum on each side of the centre, we may consider the edges of the spectral image to be defined by the position of these minima; or, in other words, consider that the apparent "width" of the line is $m_{0} m_{0}$ (fig. 1). For the value $\beta=0$ this width for an infinitely narrow slit and monochromatic radiation is of course $2 x_{0}$. The effect of an increasing absorption is to increase all the ordinates of the diffraction pattern relatively to the central one (for $\alpha=0$ ); but the ones in the neighbourhood of the first minima are increased proportionaily more than the others, so that these points of minima are gradually obliterated, and become indistinguishable for values of $\mathrm{B} b$ greater than $1 \cdot 5$. The edges of the line then become less and less sharply defined and clearly marked. Their apparent position depends somewhat on the absolute as well as the relative intensity it different parts in the diffraction pattern ; but in general they may be taken to lie near the limits $\pm m$ defined by the relations

$$
f( \pm m)=\mathrm{I}_{ \pm m}^{2} \simeq .04 \mathrm{I}_{0}^{2}
$$

$i$. e. near the points at which the intensity of illumination in the image first falls to less than 4 per cent, of the intensity at the geometrical centre.

Assuming this criterion, we have for the relation between 
Absorption on the Resolving Power of Prism Trains. 371

$\mathrm{B} b$ (or $\beta$ ) and $w$, the apparent "width," the following values (from Table I. and fig. 1):-

$$
\begin{aligned}
& \text { For } \mathrm{B} b=000 \quad w=m_{0} m_{v}=2 \cdot 00 \alpha_{0} \text {, } \\
& \mathrm{B} b=.575 w \quad=2.04 \alpha_{10}, \\
& \mathrm{~B} b=1.386 \quad w=m_{1} m_{1}=2 \cdot 16 \alpha_{i} \text {, } \\
& \mathrm{B} b=2 \cdot 197 w \quad=3 \cdot 66 \alpha_{v}, \\
& \mathrm{~B} b=2 \cdot 77 \quad w=m_{2} m_{2}=4 \cdot 8 \alpha_{v} \text {, } \\
& \mathrm{B} b=4 \cdot 61 \quad w=m_{3} m_{3}=7 \cdot 6 \alpha_{0}, \\
& \mathrm{~B} b=7.83 w \quad=13.5 \alpha_{0} \text {. }
\end{aligned}
$$

The apparent width of the line for the last value of $\mathrm{B} b$ is therefore nearly seven times as great as the theoretical width, $2 \alpha_{0}$, for zero absorption. This large increase in $w$, together with the accompanying reduction in the practical resolvingpower, $\mathbf{R}_{\beta}$, is quite sufficient to explain the apparent haziness and lack of definition frequently observed in certain portions of a spectrum formed by prisms with strong selective absorption.

The effect of absorption on the form and distribution in intensity in the spectral image, and hence on the resolvingpower of the prism-train, may be entirely eliminated by mechanical diaphragming of the prism aperture. By referring to equation (7) we see that the form of the expression for $l^{2}$ is the same as would be obtained if the illumination were uniform over the wave-front, and the latter were limited by an aperture whose length (along the $x$ axis) is $b$, as before, but whose width at any point is the ordinate to the exponential curve

$$
y=a e^{-\mathrm{B} x} . . \quad . \quad . \quad . \quad . \quad .
$$

If, therefore, we limit the aperture of the prism-train by a diaphragm whose width is $y^{-1}$, the illumination remaining the same as in (7), we obtain for the distribution in intensity in the image of the spectral line

$$
\begin{aligned}
\mathrm{I}_{\xi}^{2} & =\frac{i^{2} a^{2}}{\lambda^{2} f^{2}}\left[\int_{-\frac{b}{2}}^{+\frac{b}{2}} e^{-\mathrm{B} x} e^{\mathrm{B} x} \sin \frac{2 \pi \xi}{\lambda f^{\prime}} x d x\right]^{2} \\
& +\frac{i_{j}{ }^{2} a^{2}}{\lambda^{2} f^{2}}\left[\int_{-\frac{b}{2}}^{+\frac{b}{2}} e^{-\mathrm{B} x} e^{\mathbf{B} x} \cos \frac{2 \pi \xi}{\lambda f^{\prime}} x d x\right]^{2} \\
& =\frac{i_{0}{ }^{2} a^{2}}{\lambda^{2} f^{2}}\left[\int_{-\frac{b}{2}}^{+\frac{b}{2}} \cos \frac{2 \pi \xi}{\lambda f} x d x\right]^{2}=\frac{i_{0}{ }^{2} a^{2} b^{2}}{\lambda^{2} f^{2}} \frac{\sin ^{2} \frac{\pi \xi b}{\lambda f}}{\left(\frac{\pi \xi l}{\lambda f^{\prime}}\right)^{2}}, .
\end{aligned}
$$


which is the same as obtained with rectangular aperture of height $d$, unaffected by absorption, save that the absolute intensities at every point in the pattern are diminished in the ratio

$$
\frac{\mathrm{I}_{00}{ }^{2}}{\mathrm{I}^{2}}=\frac{i_{0}^{2} a^{2}}{d^{2}} \text {. . . . . . . . }
$$

Since the intensity in the transmitted wave-front at the thin edge of the prisin is the same as in the incident beam $(l=0)$, we have, by making $x=-\frac{l}{2}$ in $(6)$,

$$
i_{0}{ }^{2} e^{\mathrm{B} b}=1 \text {, or } \quad i_{0}{ }^{2}=e^{-\mathrm{B} b} \text {. }
$$

Also if we choose $\alpha$ in (25) such that the greatest width of the diaphragm is equal to $d$, we have

or

$$
\begin{aligned}
& d=a e^{\frac{\mathrm{B} b}{2}} \\
& a^{2}=d^{2} e^{-3 b} .
\end{aligned}
$$

Hence we have from (27), (28), and (29)

$$
\frac{\mathrm{I}_{00}^{2}}{\mathrm{I}^{2}}=e^{-\mathrm{E} u} \text {. . . . . . . . }
$$

For the Bruce spectroseope before considered the value of $\mathrm{B}$ b for wave-length 3900 is, as already computed,

$$
\begin{aligned}
\mathrm{B} b & \cong 6.59 ; \\
\therefore \quad \mathrm{I}_{00}^{2} & \simeq 000002 .
\end{aligned}
$$

Although so much light is lost in diaphragming the aperture as above described, the intensity in the resultant spectral image will compare somewhat more favourably with that secured under the actual conditions of absorption with full aperture, because the "width" of the line is so much reduced when the diaphragm is used. The intensity at the centre of the diffraction-image with full aperture $b d$ (see 10) is

$$
\mathrm{I}_{01}{ }^{2}=i_{0}{ }^{2} \frac{l^{2} d^{2}}{\lambda^{2} f^{2}} \cdot \frac{e^{\mathrm{B} b}+e^{-\mathrm{B} b}-2}{(\mathrm{~B} b)^{2}} . . .
$$

The ratio between this intensity and the intensity of the image formed by the same instrument, unaffected by absorption, will be

$$
\frac{\left.\mathrm{I}_{1}^{2}\right)^{2}}{\mathrm{I}^{2}}=\frac{e^{-\mathrm{B} b}}{\left(\mathrm{~B}()^{2}\right)^{2}}\left\{e^{\mathrm{B} b}+e^{-\mathrm{B} b}-2\right\} . \quad . \quad .
$$


Absorption on the Resolving Power of Prism Trains. $\quad 373$

For the same case considered before for which $B l$ is 6.59 , we have

$$
\frac{I_{0}^{2}}{\mathrm{I}^{2}}=023 .
$$

Finally, for the ratio between the intensities with and without a diaphragm we have from (30) and (32),

$$
\frac{\mathrm{I}_{00}{ }^{2}}{\mathrm{I}_{0}{ }^{2}}=\frac{e^{-\mathrm{B} b}(\mathrm{~B} b)^{2}}{e^{\mathrm{B} h}+e^{-\overline{\mathrm{B}} \bar{b}-2}} \text {. . . . . }
$$

which gives us for the Bruce instrument in the $\mathrm{H}$ regions

$$
\frac{\mathrm{I}_{00}{ }^{2}}{\overline{\mathrm{I}}_{0}{ }^{\underline{2}}}=.0001 . . . \quad \text {. . . . }
$$

The curve bounding the edges of the diaphragm for any given case is given by the equation [see (25) and (29)],

$$
y= \pm e^{+\beta \mathbb{N} \frac{\sin \varphi / 2}{\sqrt{1-x^{2} \sin ^{2} \phi / 2}}\left(x-\frac{b}{2}\right)} \text {. . . . }
$$

For the above case

$$
\begin{aligned}
& \mathrm{B}=\beta \mathrm{N} \frac{\sin \phi / 2}{\sqrt{1-n^{2} \sin ^{2} \phi / 2}}=1 \cdot 29, \\
& b=5 \cdot 1 .
\end{aligned}
$$

If with these values of $b$ and $\mathrm{B}$ we make $d=b$ and compute $y$ for different values of $x$, we shall find the form of the required diaphragm to be that shown in fig. 4 (Pl. VIII.). The widths at the two ends are $0.008 \mathrm{~cm}$. and $5.1 \mathrm{cms}$. respectively, with the wide end toward the base of the prism.

When so placed this diaphragm will entirely eliminate the effect of the variable absorption, but only, as indicated by (33) and (33a), at a very large sacritice of intensity of illumination. This device can therefore be used only when we have abundance of light. In other cases the only means we have of reducing the effect of absorption for any given material is to decrease the resulving-power of the prism-train either by reducing the aperture or the number of prisms, or both.

\section{General Conclusion.}

The results of the preceding investigation show that in the case of prismatic spectroscopes we are confined to certain resolving-powers. We are limited in one direction by the physical properties of the glass now at our disposal, in another by the difficulty, we might almost say impracticability, of obtaining blocks of crystalline minerals of larger than a certain size, and in a third by lack of sufficient illumination.

Phil. Mag. S. 6. Vol. 5. No. 27. Mareh 1903. $2 \mathrm{C}$ 
Our only means of obtaining more powerful instruments seems to lie, therefore, in the further development of the grating-spectroscope. The maximum resolving-powers that have thus far been attained with the latter class of instruments are some what less than 400,000 units *, about the maximum which, as we have now shown, is attainable with prismatic spectroscopes in the visible region of the spectrum. As the writer has already shown + instruments having at least three times this resolving-power are not only exceedingly desirable, but absolutely necessary to the successful investigation of certain spectral phenomena. We must therefore aim to ultimately produce gratings at least $40 \mathrm{cms}$. diameter. A machine designed to rule gratings of this size was begun by the writer seven years ago (1896), but owing to the many interruptions to which reference was made at the beginning of the article, work on it was only recently completed $\ddagger$. The construction of a machine of about the same size was begun in 1899 by Professors Michelson and Stratton at the University of Chicago ; but work on this has also been much interrupted and delayed by the resignation of Professor Stratton to accept the directorship of the new National Bureau of Standards at Washington. More recently still Jewell, at Johns Hopkins, has been remodelling one of the Rowland dividing-engines to rule $10 \mathrm{in.}(25 \mathrm{~cm}$.$) gratings.$

It is to be hoped, therefore, that through the united efforts of all of those now working on this problem, gratings of a considerably larger size than we now have may soon become a commercial possibility.

Allegheny Observatory, November, 1902.

XXXV. The Use of Contour Integration in the Problem of Diffraction by a Wedge of any Angle. By H. S. Carsiaw, Professor of Pure and Applied Mathematics in the University of Sydney, N.S.W.\$

$\mathbf{I}^{\mathrm{T}}$ N the Appendix on Diffraction to his Adams Prize Essay \| Macdonald gives a discussion of the two-dimensional

* See paper on "Resolving Power of Telescopes and Spectroscopes for Lines of Finite Width," Phil. Mag. vol, xliii. p. 320, May 1897 .

t Loc. cit. The large solar grating-spectroscopes of the new Allegheny Observatory are designed for gratings of $25 \mathrm{cms}$. and $40 \mathrm{cms}$. aperture Report of Director, 1900.

$\$$ See Report of the Director, Allegheny Observatory, 1900, p. 22. The final setting up of the new engine has been still further delayed by the delay in the completion of the new Observatory and Laboratories.

$\S$ Communicated by the Author.

i| 'Electric Wares,' by H. M. Macdonald, Camb. 1902. 

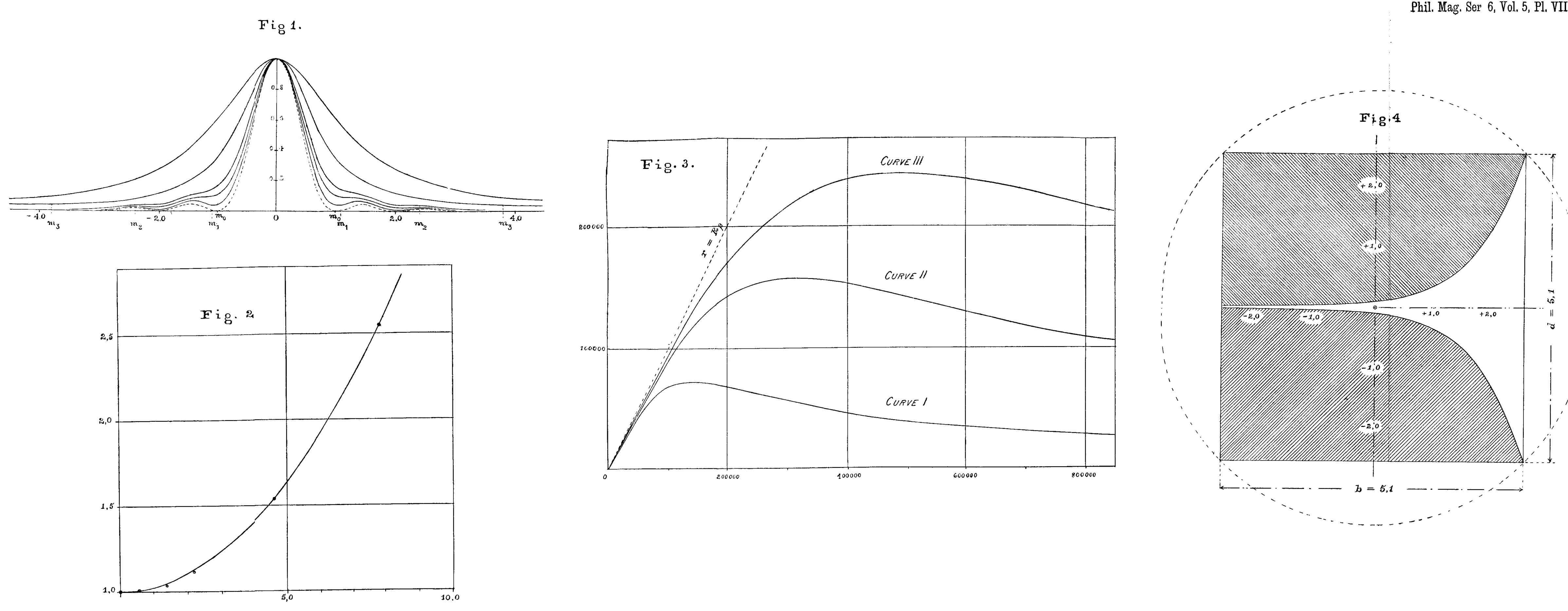